\title{
Treatment of refractory anterior knee pain using botulinum toxin type A (Dysport) injection to the distal vastus lateralis muscle: a randomised placebo controlled crossover trial
}

\author{
Barbara J Singer, ${ }^{1}$ Peter L Silbert, ${ }^{1}, 2$ Swithin Song, ${ }^{3}$ John W Dunne, ${ }^{1,2}$ Kevin P Singer ${ }^{1}$
}

${ }^{1}$ Centre for Musculoskeletal Studies, School of Surgery, The University of Western Australia, Perth, Western Australia

2Department of Neurology, Royal Perth Hospital, Perth, Western Australia

${ }^{3}$ Department of Radiology, Royal Perth Hospital, Perth, Western Australia

\section{Correspondence to}

Dr Barbara J Singer, School of Surgery, The University of Western Australia, Level 2 Medical Research Foundation Building, Royal Perth Hospital, Perth WA 6000, Western

Australia;

barbara.singer@uwa.edu.au

Accepted 19 January 2010 Published Online First 23 April 2010

\begin{abstract}
Objectives This randomised controlled crossover trial examined the efficacy of botulinum toxin type $A$ (BoNT-A) injection, plus an exercise programme, to remediate chronic anterior knee pain (AKP) associated with quadriceps muscle imbalance.
\end{abstract}

Methods 24 individuals with refractory AKP received either BoNT-A (500 U Dysport) or the same volume saline injection to the vastus lateralis (VL) muscle and performed home exercises focusing on re-training the vastus medialis (VM) muscle. All subjects were offered open-label injection at 12 weeks. Knee-related disability (anterior knee pain scale; AKPS) and activity-induced pain (10 $\mathrm{cm}$ visual analogue scale) at 12 weeks were the primary outcomes. Peak isometric extensor force was recorded and normalised VL:VM ratios were derived from simultaneous surface electromyography. Selfreported pain and disability measures were collected at six time points to a mean of $20 \pm 8$ months.

Results 14 subjects received BoNT-A and 10 placebo injection. Improvement at 12 weeks was significantly greater for BoNT-A compared with placebo-injected subjects for the AKPS ( $<<0.03)$, pain on kneeling $(p<0.004)$, squatting $(p<0.02)$ and level walking $(p<0.04)$. At week 12, five placebo subjects crossed over to open-label injection. At 24 weeks, 16 of 19 BoNT-A-injected and two of the remaining five placeboinjected subjects were either satisfied or very satisfied with treatment outcomes. Improvements were maintained in 11 of 14 BoNT-A-injected and two of five placebo subjects available at longer-term follow-up. Conclusion BoNT-A injection produced a greater reduction in pain and disability than placebo injection in carefully selected patients with chronic AKP related to quadriceps muscle imbalance.

Chronic anterior knee pain (AKP) is a disabling painful condition associated with significant activity limitation and reduced quality of life. ${ }^{1}$ It has a multifactorial aetiology with biomechanical abnormalities, anatomical variations and underlying pathology all potentially contributing. ${ }^{2} 3$ Conservative therapy may improve outcomes for some individuals with $\mathrm{AKP}^{4-6}$; however, as many as one in four patients experience chronic knee symptoms. ${ }^{7}$ Abnormal lateral tracking of the patella, associated with an imbalance in activation of the medial and lateral components of the quadriceps muscle, has been proposed as a possible contributor to AKP. ${ }^{8}$ Consequently, it is possible that treatment using botulinum toxin type A (BoNT-A) to address the imbalance between the relatively overactive vastus lateralis (VL) muscle and the less active vastus medialis (VM) may alleviate symptoms. ${ }^{9}$ Intramuscular injection of BoNT-A produces temporary presynaptic blockade of acetylcholine release, resulting in a dose-dependent weakening of muscle contraction. In animal models, restoration of muscle function has been shown to occur within 3 months of injection; initially due to the release of acetylcholine from newly formed nerve sprouts and eventually to complete recovery of the original nerve ending. ${ }^{10}$

The aim of this double blind, randomised, placebo injection controlled crossover trial was to investigate the efficacy of an intramuscular injection of BoNT-A to the distal portion of the VL muscle to provide a 'window of opportunity' to re-train the medial quadriceps and thus restore more balanced knee extensor control during functional activity. The primary outcomes were kneerelated disability and activity-induced pain at 12 weeks postinjection.

\section{MATERIALS AND METHODS}

The enrolment schedule is shown in figure 1.

All participants provided written informed consent before enrolment. The study was approved by the institutional ethics committee and listed in the Australian New Zealand Clinical Trials registry (www.anzctr.org.au) ACTRN12605000775617.

\section{Subjects and study design}

Volunteers who were $15-55$ years of age, with a history of symptoms suggestive of AKP of more than 6 months' duration, who had failed conservative care but had no previous surgical intervention, were screened by phone. Individuals who met the initial criteria attended for clinical assessment to determine eligibility. Consenting individuals underwent a screening computed tomography examination to exclude those with established patellofemoral joint degenerative change. Subjects were only included if there was clear evidence of VL muscle dominance ${ }^{11}$ assessed using surface electromyography (EMG) during isometric extension in $30^{\circ}$ of knee flexion. In individuals without knee pain, the ratio of VL:VM activation has been shown to be close to $1: 1$ during an isometric contraction at $10^{\circ}$ knee flexion..$^{12}$ Individuals were excluded if they had excessive patellofemoral joint laxity or previous patellar dislocation, body mass index greater than 30 , radiographically determined joint degeneration, der the BMJ Jour ined scheme, see http $/ /$ bjsm.bmj.com/info/unlocked.dt 


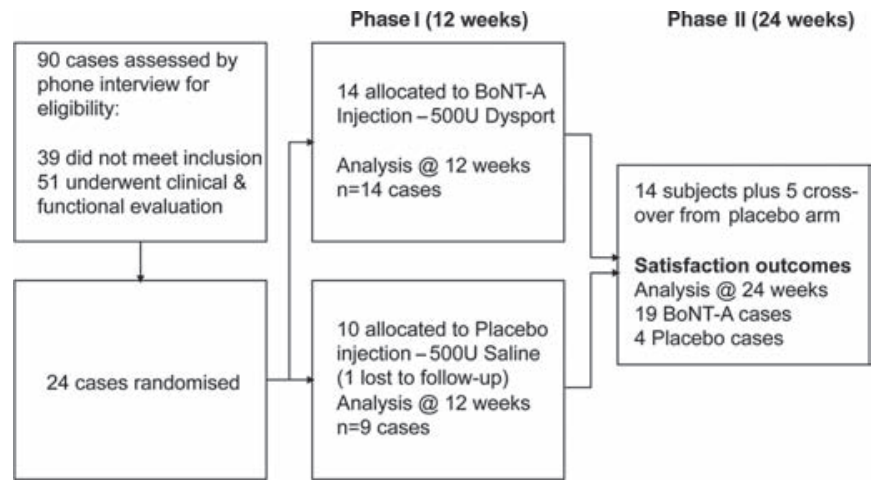

Figure 1 Study design and subject enrolment. BoNT-A, botulinum toxin type A.

or contraindications to BoNT-A injection including pregnancy. Subsequently, three baseline assessments were performed, at least 1 week apart, to establish the stability of self-report and objective measures. Following this a computer-generated random assignment was used to allocate subjects in blocks of four to BoNT-A or same volume placebo injection. Both groups undertook a daily home programme of exercises typically used in this cohort. Subjects, investigators and assessors were blinded to group allocation. At 12 weeks postinjection, all subjects had the opportunity to have an open-label BoNT-A injection if they considered that there was no change in their knee symptoms. Data were not unmasked at this time point. Subjects receiving a second injection continued with the exercise programme for a further 12 weeks. Assessments occurred at 2, 6, 12, 18 and 24 weeks from each injection. Additional self-report data were collected by mailed questionnaires up to a mean of $20 \pm 8$ months after entry to the study.

\section{Procedures}

An intramuscular injection of $500 \mathrm{U}$ Dysport (Ipsen) diluted with $4 \mathrm{ml}$ physiological saline was performed by an experienced neurologist (PLS). Needle EMG was used to guide the intramuscular injection particularly in the most distal musculotendinous portion of the VL muscle (figure 2). The dose was divided and administered over eight injection sites; with $0.5 \mathrm{ml}$ injected at each site. (figure 2). Placebo subjects received the same number of injections and volume of saline. Records of group allocation were maintained by a neurophysiology technician whose only responsibility was randomisation and drawing up the injectate.

Subjects were instructed by experienced physiotherapists (KPS, BJS) in a standard AKP exercise programme to be performed at home twice daily. Repetitions were customised according to the number the individual could perform correctly without fatigue within a session. Participants kept a diary during the 12 -week programme to record the number of exercises performed and any symptoms experienced. The diary was checked and progressions confirmed at each clinic visit.

\section{Outcome measures}

The primary outcome was the change in self-reported knee pain and disability from baseline to 12 weeks postinjection. The third baseline measure was used for all comparisons.

1. The anterior knee pain scale (AKPS) ${ }^{13}$ is a 13 -item selfreport questionnaire. The maximum score is 100. Lower scores indicate greater pain/disability. A change of 14 points

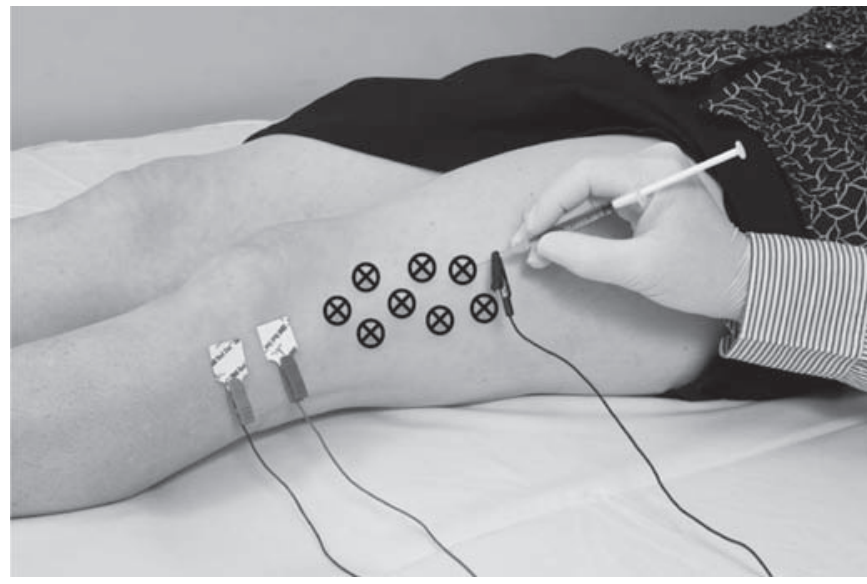

Figure 2 Intramuscular injection of botulinum toxin type A or same volume placebo was performed by an experienced injector employing electromyography guidance. Multiple injection sites were employed to ensure the spread of injectate. A model is depicted for illustrative purposes.

has previously been reported to be the minimal clinically important difference for the AKPS. ${ }^{14}$

2. Rating of pain from four potentially pain-provoking activities-ascending or descending stairs, kneeling, squatting and level walking-was recorded using a $10 \mathrm{~cm}$ visual analogue scale (VAS). ${ }^{15}$ A change of 20/100 was considered to be clinically significant. ${ }^{16}$

3. An overall rating of improvement survey (level of satisfaction with treatment outcome) was completed at 24 weeks postinjection. This questionnaire allowed participants to nominate the treatment(s) they thought they had received and whether they would have this treatment again. They were asked to rate, on a five-point Likert scale, their current status with regard to knee symptoms (swelling, stiffness, etc) and pain, restriction in participation in daily living, recreational and work activities (marked worsening to marked improvement), in addition to their satisfaction with treatment outcomes (very dissatisfied to highly satisfied).

Two additional variables were examined:

1. Peak knee extensor force during an isometric quadriceps contraction with the limb fixed in $30^{\circ}$ of flexion was assessed using an instrumented dynamometer. Following a warm-up, three trials were conducted and the best trial was used for analysis. For consistency, a standard instruction was given without encouragement during the tests.

2. Normalised VL:VM ratios were derived from surface EMG recording using the method reported by $\mathrm{Ng}$ and Cheng. ${ }^{11}$ EMG electrode pairs (3M Red-Dot; St Paul, Minnesota, USA) were applied in line with the muscle fibre direction of VM and VL, using an interelectrode distance of $25 \mathrm{~mm}$. Raw data for force and surface EMG were sampled at $1000 \mathrm{~Hz}$ and stored to disc. EMG recordings used a $6-400 \mathrm{~Hz}$ bandwidth acquired under Labview software (National Instruments Corporation, Austin, Texas, USA). A 1000 ms epoch, at the point of peak force, was used to extract the EMG signals, which were full-wave rectified and root mean square processed. Force and ratio data at baseline were compared with each time point to 12 weeks postinjection.

Qualitative data were collected from the subjects' diaries to describe any adverse effects, compliance with the home 

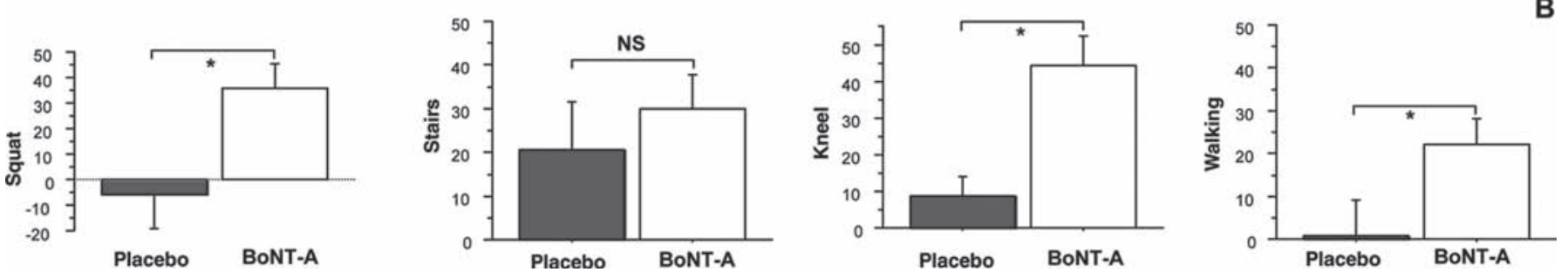

Figure 3 The mean $( \pm S E)$ change between baseline and 12 weeks post-injection for self-reported knee-related disability $(A)$ and activityinduced pain (B) is depicted for botulinum toxin type A (BoNT-A)-injected and placebo groups. Statistically significant differences * were seen between groups for four of five parameters measured, favouring the BoNT-A-injected group. AKPS, anterior knee pain scale.

programme, as well as any change in physical activity levels during the course of the study.

\section{Statistical analysis}

An intention to treat analysis was performed. The groups were examined for comparability of demographics and self-reported pain and disability data at baseline using independent t tests. Repeated measures analysis of variance (StatView) was used for planned comparisons (Scheffe) to assess the within-group change over time for knee force and EMG ratios; whereas unpaired t tests of the change scores were used for betweengroup comparisons. A probability of $\mathrm{p}<0.05$ was adopted for all statistical tests.

\section{RESULTS}

Study enrolment is summarised in figure 1. Twenty-four individuals met the entry criteria. Subjects had an average duration of symptoms of 5.7 years (range 1-10). Many had sought a surgical opinion and five cases were wait-listed for surgical management.

In phase I, 14 individuals received Dysport (eight women, six men; mean age 31.5 years (range $15-48$ ), duration of symptoms 66 months) and 10 received placebo injection (nine women, one man; mean age 27.4 years (range 18-44), duration of symptoms 80 months). One placebo subject dropped out of the study at 8 weeks due to work-related relocation and was lost to follow-up.

There were no statistically significant differences between the groups in any of the measured variables at baseline except for pain on squatting (figure 3 ). For this variable, the BoNT-A group reported a mean pain score of $60.6 / 100$, whereas the placebo group mean score was $40.3 / 100$ ( $\mathrm{p}<0.001$ ). Participants reported mean baseline AKPS scores of 65/100 (BoNT-A group) and 69/100 (placebo group), which implies moderate pain and disability. ${ }^{17}$ All subjects reported having significantly reduced their involvement in pain-provoking physical activity in order to control their symptoms.

Reported compliance with the home exercise programme was excellent for all subjects and $93 \%$ of all scheduled follow-up appointments to 6 months postinjection were attended.
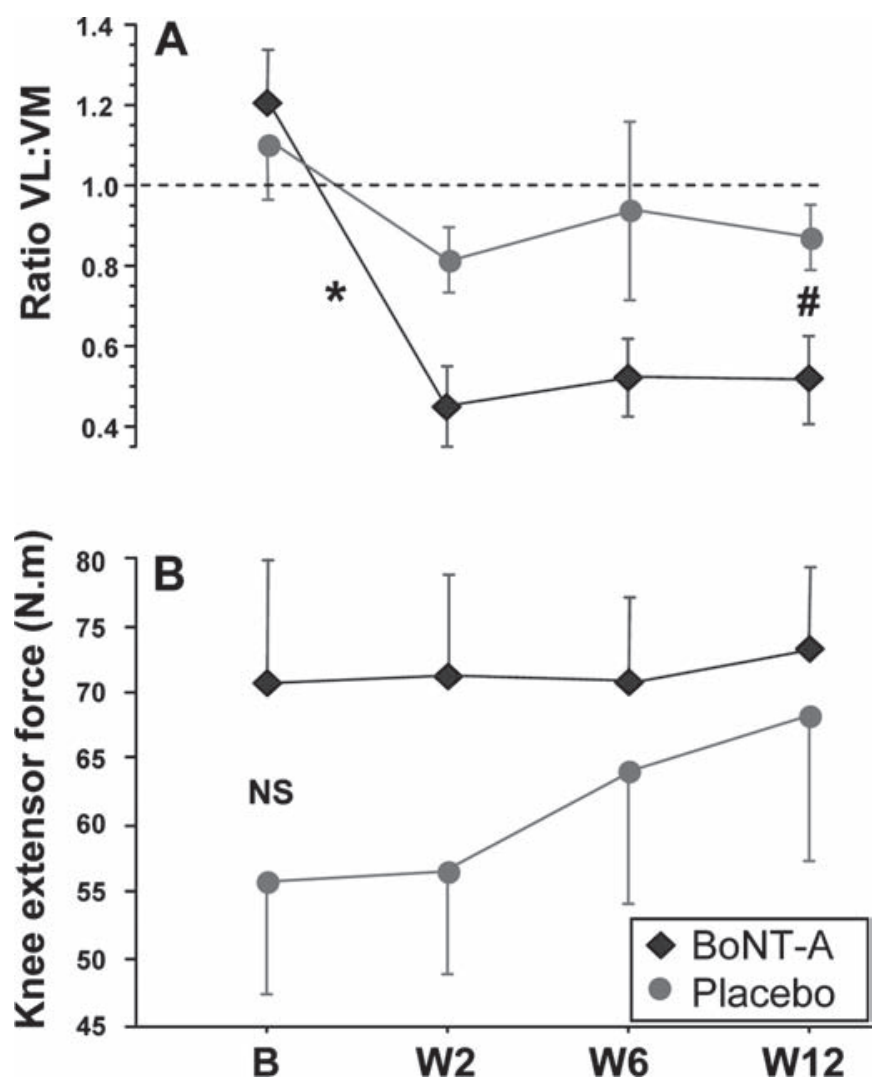

Figure 4 (A) A significant change in vastus medialis (VM) activation in the botulinum toxin type $A$ (BoNT-A)-injected group only $\left({ }^{*}\right)$, during static quadriceps contraction at $30^{\circ}$ knee flexion, occurred by week 2 (W2), which was sustained at week 12 (\#). The dotted line represents parity of vastus lateralis (VL):VM activation. (B) There was no change in the mean $( \pm S E)$ peak knee extensor force between BoNT-A-injected and placebo groups, or for the effect of time. B, baseline; W, week.

\section{Phase I (12-week comparison)}

Data are presented in figures 3 and 4 .

1. Improvement between baseline and 12 weeks was significantly greater for BoNT-A than placebo-injected subjects for the AKPS $(p<0.03)$ (figure $3 \mathrm{~A}$ ), pain on kneeling 
$(p<0.004)$, squatting $(p<0.02)$ and level walking $(p<0.04)$ (figure $3 \mathrm{~B}$ ).

2. Change in AKPS scores exceeded the minimal clinically important difference ${ }^{14}$ in the BoNT-A-injected subjects only (mean increase 15 points, $\mathrm{p}<0.001$ ) (figure $3 \mathrm{~A}$ ).

3. The BoNT-A-injected group demonstrated a clinically significant change ${ }^{16}$ in mean pain scores for kneeling $(-50.5$, $\mathrm{p}<0.001)$, stair walking $(-20.9, \mathrm{p}<0.006)$, squatting $(-30.8$, $\mathrm{p}<0.001)$ and level walking $(-20.3, \mathrm{p}<0.003)$. In placebo subjects, there was a reduction for stair walking only (-20.4); however, this was not a statistically significant change from baseline $(p<0.097)$ (figure 3B).

4. A dominant pattern of VL activation was present at baseline in all subjects ${ }^{11} 12$ (figure $4 \mathrm{~A}$ ). The normalised ratio of VM:VL amplitude during isometric quadriceps contraction was reversed at week 2 , and remained significantly different to baseline in the BoNT-A-injected group at all timepoints postinjection (figure $4 \mathrm{~A})(\mathrm{p}<0.001)$. Ratios in the placeboinjected subjects also became closer to $1: 1$ but the difference from baseline was not significant at any time point postinjection (figure 4A).

5. There was a small improvement in peak knee extensor force over time in the placebo group but not in the BoNTA-injected group (figure 4B). The difference between groups was not significant at any time point.

\section{Phase II (crossover and long-term follow-up)}

At 12 weeks postinjection, five subjects elected to receive an open-label injection of Dysport on the basis that their symptoms were unchanged. Self-reported pain and knee-related disability for these crossover subjects showed improvement beyond the second baseline (figure 5). Statistical analysis was not performed due to the small number of subjects in the crossover group. When group allocation was unmasked at 24 weeks, all five crossover subjects were found to have initially received placebo injection. An additional four subjects did not consider that they had made any improvement at 12 weeks but elected not to crossover due to travel or study commitments. Two of these individuals had received Dysport injection in phase I.

At 24 weeks after the initial injection all subjects completed an exit questionnaire. Twenty of 24 subjects correctly identified their group allocation. Two placebo-injected subjects thought that they had received the active drug injection and two BoNT-A-injected subjects were unsure if they had received the active treatment.

At 24 weeks, 16 out of 19 BoNT-A-treated subjects reported that they were highly satisfied (8/19) or satisfied (8/19), whereas three of 19 were dissatisfied with treatment outcomes. The reasons for dissatisfaction included ongoing activity-induced pain (two subjects) and a perception of asymmetry of the distal aspect of the quadriceps muscle (one subject). One of the placebo-only subjects was highly satisfied, one was satisfied and the remaining placebo-injected subjects were dissatisfied with treatment outcomes due to persistent or worsening pain.

Long-term data for pain (VAS) and knee-related disability (AKPS) were available for 17 of the 24 subjects enrolled in the study (with a mean follow-up of $20 \pm 8$ months from injection). Sustained improvement was reported in 11 of 14 BoNTA-injected subjects. One BoNT-A-injected subject elected to have knee surgery at 14 months postenrolment, and two had regressed to close to baseline by 12 months. Two of the five placebo-only subjects had maintained the improvements observed at 6 months, one had regressed to worse than baseline and two were lost to follow-up.
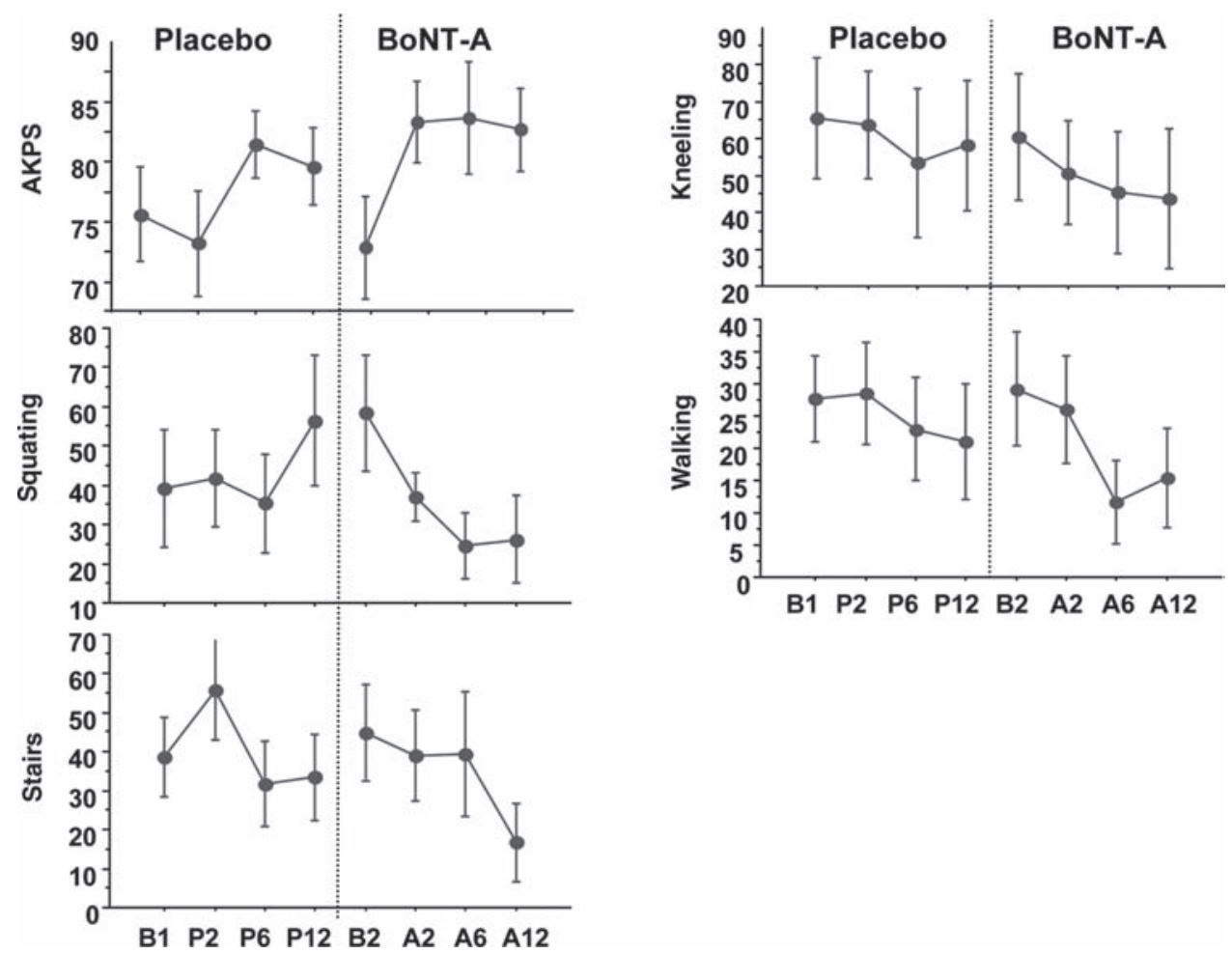

Figure 5 Data for the crossover subgroup $(n=5)$ for self-reported knee-related disability (anterior knee pain scale; AKPS) and activity induced pain (visual analogue scale) for four pain-provoking tasks taken during the initial 12-week placebo phase. The subsequent post-botulinum toxin type A (BoNT-A) injection phase resulted in a further improvement from the second baseline (B2). 


\section{Adverse effects}

Few adverse effects were reported, and all related to slight bruising or injection-related pain, which resolved within days. Slight distal thigh asymmetry, which persisted to at least 24 weeks postinjection, was present in six BoNT-A-injected subjects. Only one subject perceived this to be an adverse outcome.

\section{DISCUSSION}

The aim of this randomised controlled trial was to examine the effect of the intramuscular injection of BoNT-A (Dysport) to the distal portion of the VL muscle, in addition to a standard home exercise programme, to remediate longstanding AKP in carefully selected subjects with demonstrated VL muscle dominance. The group studied had an average duration of knee symptoms of 5.7 years (range 1-10); and had exhausted conservative management, including having previously performed similar exercises to those that were undertaken as part of the study. Data presented support the hypothesis that this novel intervention has the potential to induce improvements in longstanding knee pain and disability. Importantly, these improvements outlasted the 12 weeks expected duration of the drug effect based on animal studies. ${ }^{10}$

The mechanisms underlying these improvements may be partly elucidated from the present study. The ratio of VM to VL muscle activation during isometric knee extension was reversed following BoNT-A injection. That is, as early as 2 weeks postinjection, the VM showed the greatest activation during isometric knee extension; whereas previously the VL was dominant. In the placebo group, the ratio of VM to VL muscle activation also changed, although to a much lesser extent, suggesting that the injection itself had some influence on muscle activation, which persisted for at least 12 weeks (figure 4A). However, in contrast to the BoNT-Ainjected subjects, the VM:VL ratio in placebo subjects was not significantly different from baseline at any time point (figure 4A). There was a steady improvement in knee extensor force production over the 12 -week period in placebo subjects (figure 4B). Peak force, which might be expected to have reduced in BoNT-A-injected subjects as a result of relative weakening of the distal VL muscle, was maintained (figure 4B). Some subjects noted an ability to recruit and sustain a contraction of the VM muscle within a week of the injection; something that they had been unable to achieve previously, despite, in some cases, electrical muscle stimulation and EMG biofeedback training. It is acknowledged that this may have led them to conclude that they had received the active treatment, with the potential for bias of subsequent self-reports; however, it is difficult to accept that this would have influenced quadriceps muscle activation ratios, which are not under volitional control. ${ }^{18}$

\section{Limitations of the study}

The relatively small numbers recruited for this study, as well as the specific inclusion and exclusion criteria used, mean that the sample is not broadly representative of all individuals with chronic AKP. The groups were well matched apart from gender allocation. The greater incidence of AKP in women is well described ${ }^{19}{ }^{20}$; however, it is possible that the gender inequity between groups may have confounded the results, particularly with respect to gender-based differences in strength and motor control. ${ }^{21}$ Future studies are needed to explore this issue.
It is well accepted that quadriceps muscle imbalance is only one of many factors contributing to AKP. ${ }^{2}{ }^{3}$ Indeed, only 26 of the 51 cases assessed, all of whom presented with symptoms suggestive of $\mathrm{AKP},{ }^{1}$ were found to have VL dominance on surface EMG evaluation. However, the present cohort represents a group who are frequently considered for surgery. Cases with refractory AKP associated with maltracking may undergo surgical procedures such as lateral retinacular release and tibial tubercle alignment. The evidence that patella malalignment is the primary cause of AKP is limited and is based on measures with poor reliability and/or validity. ${ }^{22}$ Given the lack of evidence for a clear relationship between patellofemoral joint alignment and symptoms, it could be speculated that surgical correction may not be the optimal approach to alleviate symptoms of AKP. Botulinum toxin injection is a safe, reversible treatment that does not interfere with patellofemoral joint integrity. In combination with careful assessment and management of contributing factors, BoNT-A injection has the potential to be a cost-effective treatment for carefully selected AKP patients, who are refractory to conservative management. In some cases, BoNT-A injection may alleviate the need for surgery. Further investigation of the utility of BoNT-A in larger cohorts with refractory $\mathrm{AKP}$, including patients wait-listed for surgery, and with a longer follow-up, would appear to be merited.

From our investigation, it could be speculated that the injection of BoNT-A into the distal VL muscle improved neuromotor control of the quadriceps, which facilitated normal patellofemoral mechanics. Draper et a ${ }^{23}$ have reported the utility of open magnetic resonance imaging and a semi-erect dynamic knee flexion protocol to demonstrate abnormal patellofemoral joint kinematics in individuals with AKP. In future studies it would be valuable to examine changes in physiologically loaded patellofemoral joint kinematics as an outcome of BoNT-A treatment of AKP, in addition to selfreported pain and disability.

\section{CONCLUSION}

This study extends our previous open-label study examining the efficacy of a focal injection of BoNT-A for individuals with refractory AKP associated with quadriceps muscle imbalance. ${ }^{9}$ The improvement between baseline and 12 weeks was significantly greater for BoNT-A than placebo-injected subjects for the AKPS, pain on kneeling, squatting and level walking. BoNT-A injection was associated with improved activation of VM muscle without compromise to isometric knee extensor force production. Individuals who received Dysport either as an initial or crossover treatment were generally satisfied or highly satisfied with outcomes at 6 months postinjection. Improvements were maintained in the majority of BoNT-A-injected subjects available for follow-up to a mean of 20 months.

Acknowledgements The authors acknowledge the assistance with data collection by clinic staff and neurophysiology technicians in the Department of Neurology and computed tomography radiographers at Royal Perth Hospital, and the study subjects for their compliance.

Funding Raine Medical Research Foundation at The University of Western Australia provided some funding support to the principal author (BJS); Ipsen supplied Dysport to Royal Perth Hospital at no cost and provided financial support for computed tomography costs.

Competing interests KPS and BJS have received travel support from Ipsen.

Ethics approval The study was approved by the institutional ethics committee and listed in the Australian New Zealand Clinical Trials registry (www.anzctr.org.au) ACTRN12605000775617.

Patient consent Obtained.

Provenance and peer review Not commissioned, externally peer reviewed. 


\section{What is already known on this topic}

AKP is prevalent in active individuals, more frequently noted in women.

- Patella maltracking secondary to disturbed quadriceps function is commonly assumed to contribute to this problem.

- As a result of a constellation of diagnoses for AKP, a diverse range of conservative and surgical treatment alternatives exist, many without sound evidence for efficacy.

\section{What this study adds}

- A single injection of BoNT-A, plus a conventional exercise programme, may provide a conservative treatment option to restore quadriceps muscle balance in individuals with refractory AKP.

- This study provides support for a novel application of BoNT-A to improve neuromotor control of the knee extensor mechanism and alleviate chronic AKP.

\section{REFERENCES}

1. Harrison E, Magee D, Quinney H. Development of a clinical tool and patient questionnaire for evaluation of patellofemoral pain syndrome patients. Clin $\mathrm{J}$ Sport Med 1996;6:163-70.

2. Dye SF. The pathophysiology of patellofemoral pain: a tissue homeostasis perspective. Clin Orthop Relat Res 2005;436:100-10.

3. Dixit S, DiFiori JP, Burton M, et al. Management of patellofemoral pain syndrome. Am Fam Physician 2007;75:194-202.

4. Crossley K, Bennell K, Green S, et al. Physical therapy for patellofemoral pain: a randomized, double-blinded, placebo-controlled trial. Am J Sports Med 2002:30:857-65.

5. Fagan V, Delahunt E. Patellofemoral pain syndrome: a review on the associated neuromuscular deficits and current treatment options. Br J Sports Med 2008;42:789-95

6. Collins N, Crossley K, Beller E, et al. Foot orthoses and physiotherapy in the treatment of patellofemoral pain syndrome: randomised clinical trial. Br J Sports Med 2009;43:169-71.
7. Nimon G, Murray D, Sandow M, et al. Natural history of anterior knee pain: a 14- to 20-year follow-up of nonoperative management. J Pediatr Orthop 1998:18:118-22.

8. Powers CM. Patellar kinematics, part I: The influence of vastus muscle activity in subjects with and without patellofemoral pain. Phys Ther 2000;80:956-64.

9. Singer BJ, Silbert PL, Dunne JW, et al. An open label pilot investigation of the efficacy of botulinum toxin type $A$ [Dysport] injection in the rehabilitation of chronic anterior knee pain. Disabil Rehabil 2006;28:707-13.

10. de Paiva A, Meunier FA, Molgó J, et al. Functional repair of motor endplates after botulinum neurotoxin type A poisoning: biphasic switch of synaptic activity between nerve sprouts and their parent terminals. Proc Natl Acad Sci USA 1999;96:3200-5

11. $\mathbf{N g ~ G Y , ~ C h e n g ~ J M . ~ T h e ~ e f f e c t s ~ o f ~ p a t e l l a r ~ t a p i n g ~ o n ~ p a i n ~ a n d ~ n e u r o m u s c u l a r ~}$ performance in subjects with patellofemoral pain syndrome. Clin Rehabil 2002:16:821-7.

12. Souza DR, Gross MT. Comparison of vastus medialis obliquus:vastus lateralis muscle integrated electromyographic ratios between healthy subjects and patients with patellofemoral pain. Phys Ther 1991;71:310-16; discussion 317-20.

13. Kujala UM, Jaakkola LH, Koskinen SK, et al. Scoring of patellofemoral disorders. Arthroscopy 1993;9:159-63.

14. Watson CJ, Propps M, Ratner J, et al. Reliability and responsiveness of the lower extremity functional scale and the anterior knee pain scale in patients with anterior knee pain. J Orthop Sports Phys Ther 2005;35:136-46.

15. Laprade JA, Culham EG. A self-administered pain severity scale for patellofemoral pain syndrome. Clin Rehabil 2002;16:780-8.

16. Farrar JT, Young JP,Jr LaMoreaux L, et al. Clinical importance of changes in chronic pain intensity measured on an 11-point numerical pain rating scale. Pain 2001;94:149-58

17. Crossley KM, Bennell KL, Cowan SM, et al. Analysis of outcome measures for persons with patellofemoral pain: which are reliable and valid? Arch Phys Med Rehabil 2004;85:815-22.

18. Smith T0, Bowyer D, Dixon J, et al. Can vastus medialis oblique be preferentially activated? A systematic review of electromyographic studies. Physiother Theory Pract 2009;25:69-98.

19. Boling M, Padua D, Marshall S, et al. Gender differences in the incidence and prevalence of patellofemoral pain syndrome. Scand J Med Sci Sports. Published Online First: 17 Sept 2009. doi: 10.1111/j.1600-8338.2009.00996.x

20. Taunton JE, Ryan MB, Clement DB, et al. A retrospective case-control analysis of 2002 running injuries. Br J Sports Med 2002;36:95-101.

21. Hewett TE, Myer GD, Ford KR. Decrease in neuromuscular control about the knee with maturation in female athletes. J Bone Joint Surg Am 2004; 86-A:1601-8.

22. Wilson $\mathbf{T}$. The measurement of patellar alignment in patellofemoral pain syndrome: are we confusing assumptions with evidence? J Orthop Sports Phys Ther 2007;37:330-41.

23. Draper CE, Besier TF, Santos JM, et al. Using real-time MRI to quantify altered joint kinematics in subjects with patellofemoral pain and to evaluate the effects of a patellar brace or sleeve on joint motion. J Orthop Res 2009;27:571-7. 